\author{
INEEL/CON-04-01881 \\ PREPRINT \\ Laser Acoustic Imaging of Film Bulk Acoustic \\ Resonator (FBAR) Lateral Mode Dispersion
}

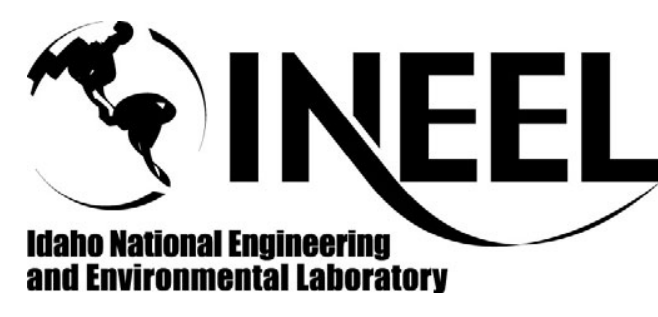

Ken L. Telschow

July $25-30,2004$

$31^{\text {st }}$ Annual Reviews of Progress in Quantitative Nondestructive Evaluation

This is a preprint of a paper intended for publication in a journal or proceedings. Since changes may be made before publication, this preprint should not be cited or reproduced without permission of the author.

This document was prepared as a account of work sponsored by an agency of the United States Government. Neither the United States Government nor any agency thereof, or any of their employees, makes any warranty, expressed or implied, or assumes any legal liability or responsibility for any third party's use, or the results of such use, of any information, apparatus, product or process disclosed in this report, or represents that its use by such third party would not infringe privately owned rights. The views expressed in this paper are not necessarily those of the U.S. Government or the sponsoring agency. 


\title{
LASER ACOUSTIC IMAGING OF FILM BULK ACOUSTIC RESONATOR (FBAR) LATERAL MODE DISPERSION
}

\author{
Ken L. Telschow \\ Physics Group, Idaho National Engineering and Environmental Laboratory \\ Idaho Falls, ID 83415-2209, USA (telsch@inel.gov)
}

\begin{abstract}
A laser acoustic imaging microscope has been developed that measures acoustic motion with high spatial resolution without scanning. Images are recorded at normal video frame rates and heterodyne principles are used to allow operation at any frequency from $\mathrm{Hz}$ to $\mathrm{GHz}$. Fourier transformation of the acoustic amplitude and phase displacement images provides a direct quantitative determination of excited mode wavenumbers at any frequency. Results are presented at frequencies near the first longitudinal thickness mode $(\sim 900 \mathrm{MHz})$ demonstrating simultaneous excitation of lateral modes with nonzero wavenumbers in an electrically driven AlN thin film acoustic resonator. Images combined at several frequencies form a direct visualization of lateral mode dispersion relations for the device under test allowing mode identification and a direct measure of specific lateral mode properties. Discussion and analysis of the results are presented in comparison with plate wave modeling of these devices taking account for material anisotropy and multilayer films.
\end{abstract}

\section{INTRODUCTION}

Microscopic film bulk acoustic resonators (FBARs) are utilized in the telecommunications industry for a variety of signal processing and routing tasks. Direct measurements of the acoustic motion of these resonators provide additional characterization of the device's electrical operation. A new method for full-field viewing these acoustic motions at $\mathrm{GHz}$ frequencies, without scanning, has been described previously [1-3]. This approach performs optical full image detection of the acoustic displacement amplitude and phase motion in a microscopic geometry using photorefractive dynamic holography. The apparatus and operation of this approach have been previously described for measuring UHF acoustical motion at a single point [2] and imaging [3,4]. In this paper we describe results and analysis from imaging measurements of the lateral acoustic mode motion in a nearly square FBAR around its series resonance and compare with model predictions.

The FBAR resonator (designated FBAR12_left) is a nearly square $160 \mu \mathrm{m}$ micromachined thin plate of Aluminum Nitride (AlN) coated with thin Molybdenum (Mo) metal electrodes exhibiting a series resonance at $875 \mathrm{MHz}$ [5], see figure 1. The thickness of the Mo layers was $0.78 \mu \mathrm{m}$ (oriented with the (111) plane normal to the thickness direction) and the AlN layer $2.24 \mu \mathrm{m}$ (0001), for a total plate thickness of $3.80 \mu \mathrm{m}$. The AlN plate is grown on a silicon wafer, etched and supported at the edges to provide stressfree boundary conditions. In operation, the FBAR vibrates primarily in the plate thickness 


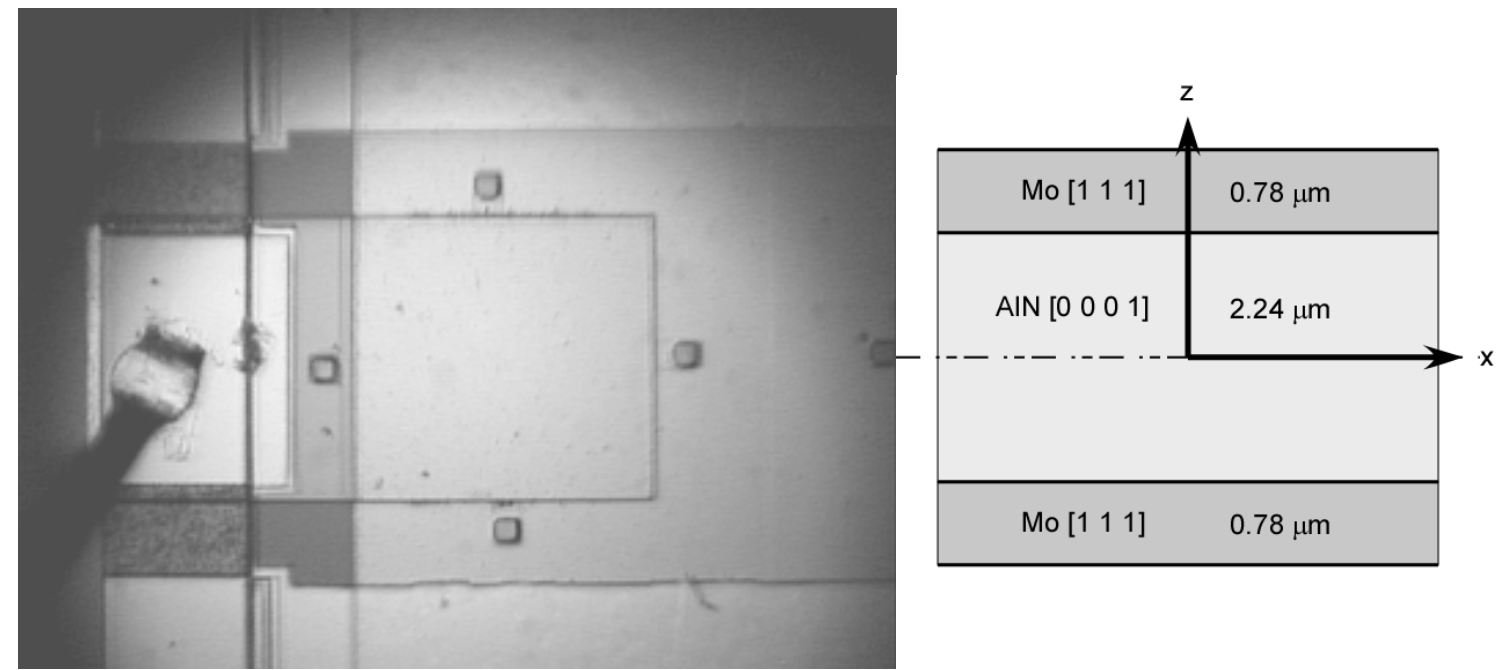

FIGURE 1. Video image of the FBAR (left) and a schematic of the layers with their orientation and thickness (right).

mode at the series resonance frequency, but also exhibits many lateral mode motions at nearby frequencies that can affect device performance. This paper describes work that identifies the lateral plate wave motions observed in the finite sized resonator through images of the motion as well as through dispersion properties of the various modes.

\section{IMAGING MEASUREMENTS}

Light from a long coherence length $532 \mathrm{~nm}$ laser was reflected from the resonator surface and combined interferometrically with a reference beam in a photorefractive crystal. The resulting diffraction grating produced images of the surface displacement at synchronized acoustic phase intervals. Operation at any frequency was achieved by tuning an electro-optic modulator to that frequency offset by $1 / 4$ of the imaging capture frequency
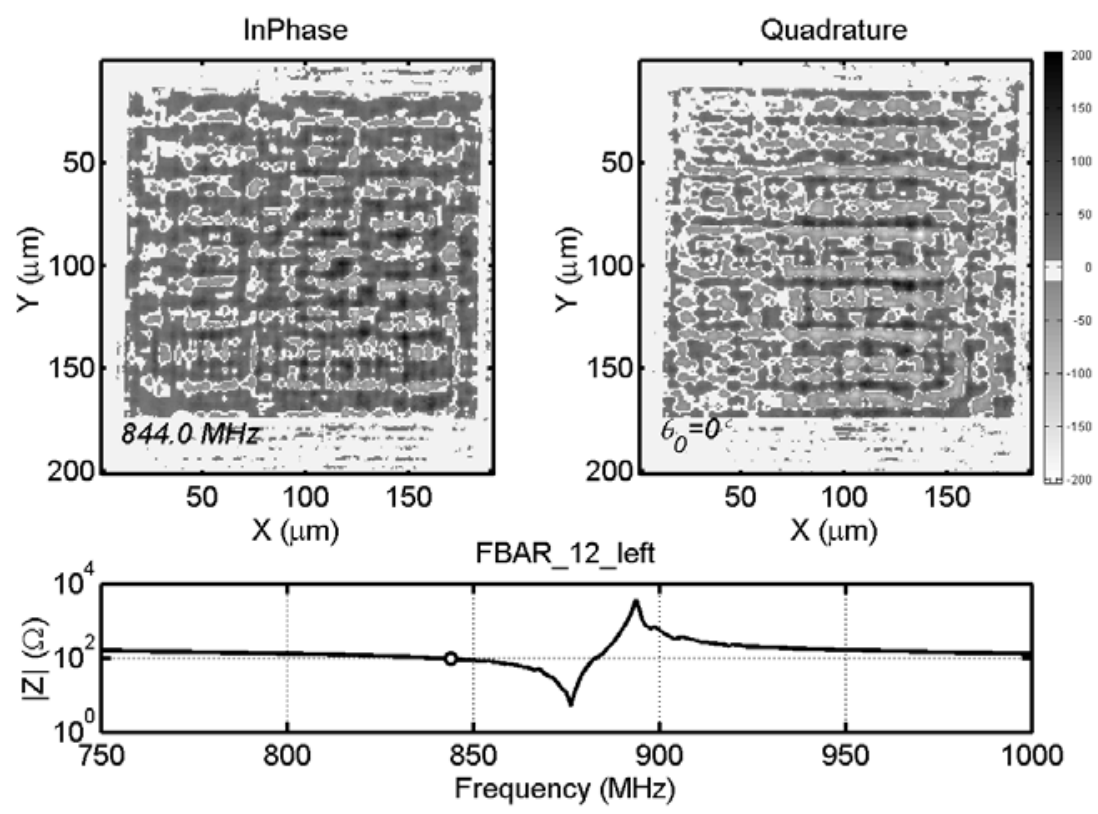

FIGURE 2. Measured in-phase and quadrature acoustic displacement at $844 \mathrm{MHz}$ along with the FBAR electrical input impedance showing a primary resonance at $875 \mathrm{MHz}$. 
of the video camera. A sequence of 4 images phase shifted by $90^{\circ}$ was recorded at a rate of $5 \mathrm{~Hz}$ (video camera running at $20 \mathrm{~Hz}$ ). Images were subtracted from their $180^{\circ}$ counterparts to produce both the InPhase and Quadrature images of the acoustic motion, allowing complete determination of acoustic normal displacement amplitude and phase at all points on the surface and in the immediate vicinity of the FBAR on the substrate. Complete data acquisition at one frequency of 256x240 pixel images (61440 spatial points) with 128 averages was achieved in less than 30 seconds providing rapid acoustical imaging.

Figure 2 shows the imaging results at a frequency just below the series resonance. The figure shows the InPhase and Quadrature acoustic displacement images that depict several patterns with different spatial wavelengths. The electrical impedance of the resonator is also shown along with a marker at the operation frequency. Both positive and negative displacements are represented in the figure with the scale shown at the right. Values near zero displacement are arbitrarily reproduced as white so that the sample outline is readily visible. As shown in this image, more than one spatial wavelength was observed.

The Fourier Transform image, shown in figure 3, shows that there are modes with four different wavelengths, all excited simultaneously. The spatial dimension was determined by calibrating the displacement image with known lateral translations from an $\mathrm{XY}$ micrometer stage (precision of $+/-0.1 \mu \mathrm{m}$ ). A striking feature, seen in the FT images, is that the response of all modes exhibits circular symmetry with respect to the XY plane. The FBAR fabrication process is designed to produce AIN in the hexagonal (Wurtzite) phase with the c-axis perpendicular to the plate surface. Materials with hexagonal symmetry exhibit isotropic symmetry of all mode wave speeds in the XY plane [6]. However, the Mo layers exhibit cubic symmetry in the (111) plane, but the AIN layer symmetry dominates the overall symmetry observed.

\section{LATERAL MODE ANALYSIS}

Since the wave speeds are symmetric in the XY plane, the FT results can be averaged with respect to angle and then plotted as a function of wavenumber, as shown in figure 3. Each of the four prominent modes is exhibited in the figure. Continuing this process for all the frequencies measured in the range from $750 \mathrm{MHz}$ to $1000 \mathrm{MHz}$ provides a visual
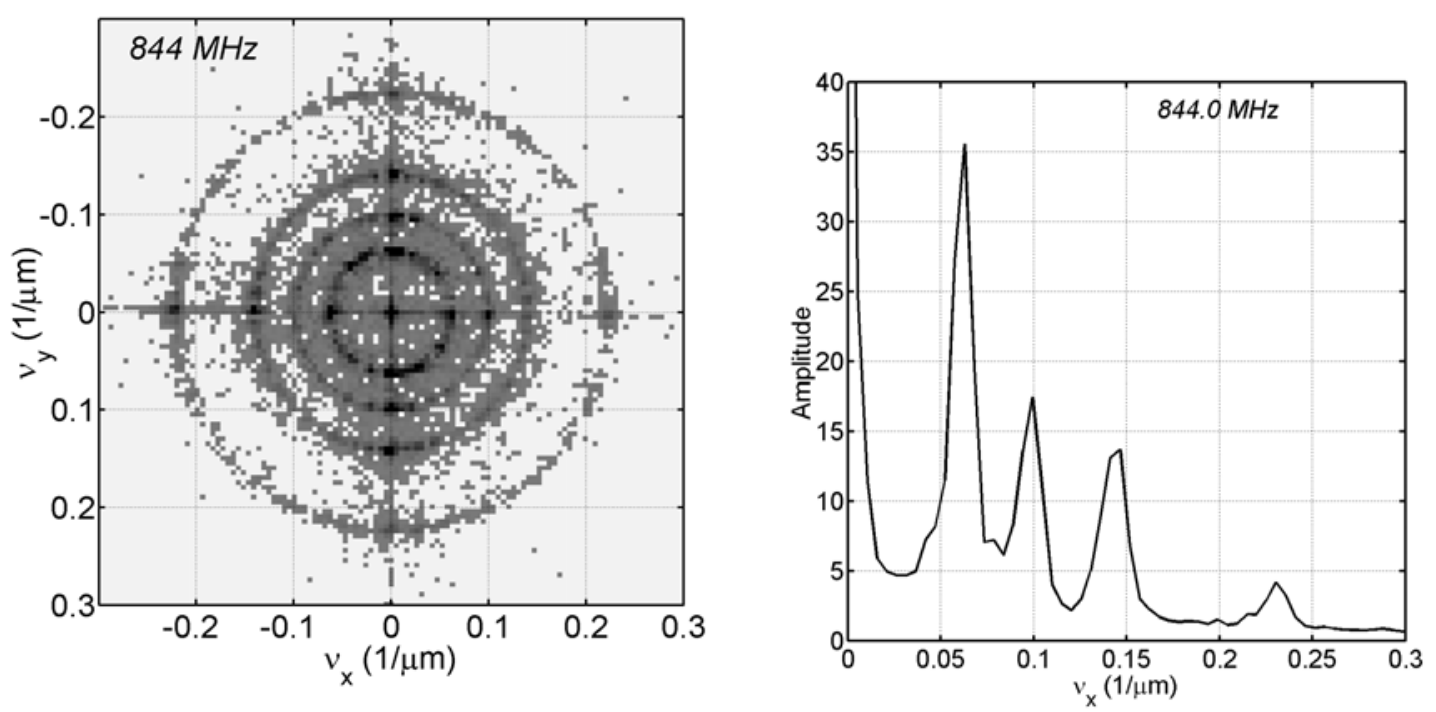

FIGURE 3. FT magnitude image and projection along the $x$-axis for a single frequency averaged over all $\mathrm{XY}$ planar angles. 


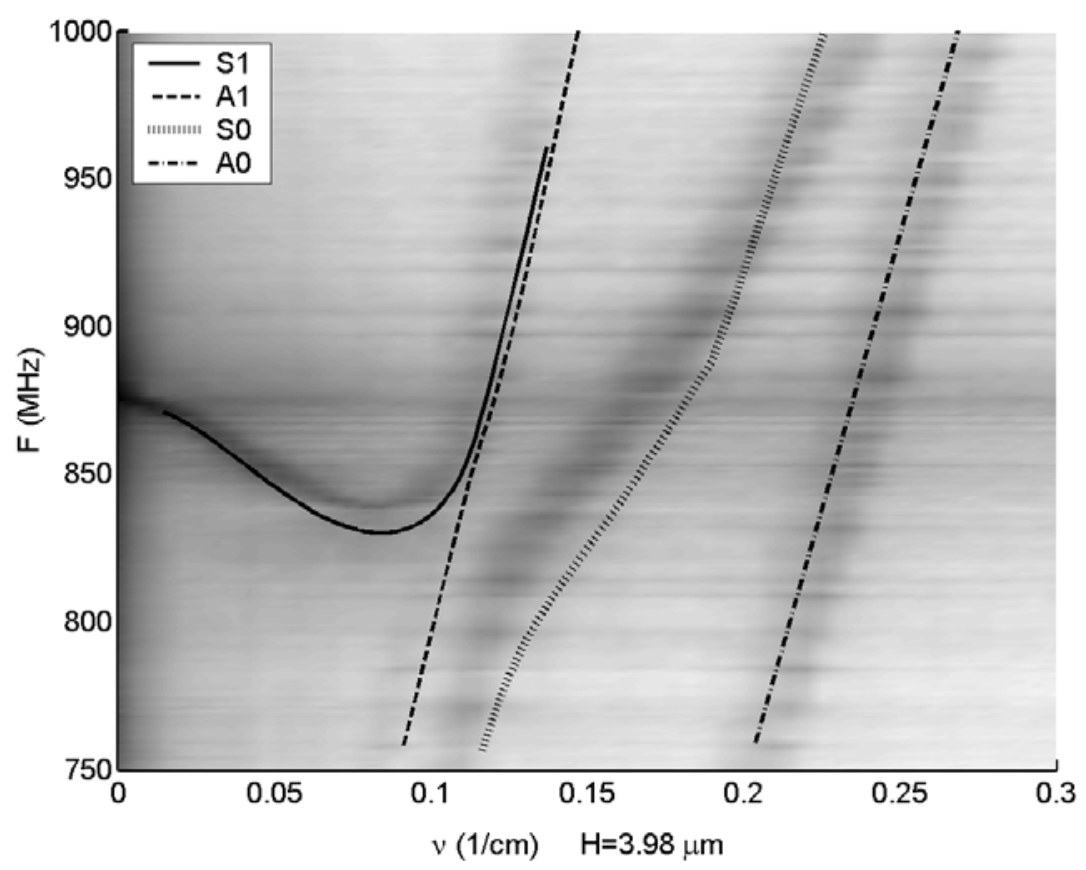

FIGURE 4. Lateral wave mode dispersion from the FFT images and calculation of the first four plate wave modes in the layered Mo/AlN/Mo plate of total thickness $3.98 \mu \mathrm{m}$.

mapping of the mode dispersion [7], as shown in figure 4. The existence of these modes comes from the resonator being finite in size and the motion from all the modes excited is needed to satisfy the boundary conditions. It is evident from the figure that several modes are present that change wavelength $(\lambda)$ as the frequency increases. In particular, the motion at $765 \mathrm{MHz}$ exhibits 3 modes that generally increase in wavenumber $(v=1 / \lambda)$ with frequency. Abruptly, at about $840 \mathrm{MHz}$ a new very strong mode appears with a wavenumber of about $0.08(1 / \mu \mathrm{m})$. This mode then splits into two parts as the frequency increases, one that decreases in wavenumber and eventually becomes the series resonance (875 MHz) with zero wavenumber and the second that increases in wavenumber similar to the other modes. Well above the series resonance, (e.g. $882 \mathrm{MHz}$ ) three distinct modes are again seen with nonzero wavenumber.

The identity of these modes becomes apparent when the dispersion curve for plate waves in anisotropic Mo/AlN/Mo plates is calculated. A calculation of the wave mode dispersion relation for the multilayered plate was performed using partial wave analysis. This method orients the material so that the given crystalline plane is normal to the plate surface, then the three elastic wave modes are found that have a phase velocity in the planar propagation direction, [001], equal to a given value for a given frequency. Linear combinations of these modes are formed that then match the boundary conditions of continuity of the displacement-traction vector at the layer interfaces. This approach is repeated for all three layers and the stress-free boundary condition at the outer surfaces is inserted resulting in a set of linear equations that must be solved simultaneously. The dispersion relations are then the values of frequency and wavenumber that set the determinant of the global matrix equal to zero, as described in [8] and references therein. This method allows use of any material for which the entire elastic stiffness matrix and orientation is known and for any planar propagation direction.

Figure 4 shows a calculation of the two lowest symmetric (S0, S1) and antisymmetric (A0, A1) modes in the Mo/AlN/Mo plate, AlN (not including piezoelectricity) in the Wurtzite structure with the c-axis perpendicular to the plate surface. The elastic 
coefficients $\mathrm{C}_{\mathrm{IJ}}$ and density $\rho$ used for this structure are depicted in table 1 , the AlN data were taken from reference [9] and that for Mo from reference [10].

\begin{tabular}{|l|l|l|l|l|l|l|l|l|}
\hline & plane & $(\mu \mathrm{m})$ & $\rho\left(\mathrm{Kg} / \mathrm{m}^{3}\right)$ & $\mathrm{C}_{11}(\mathrm{GPa})$ & $\mathrm{C}_{12}(\mathrm{GPa})$ & $\mathrm{C}_{13}(\mathrm{GPa})$ & $\mathrm{C}_{33}(\mathrm{GPa})$ & $\mathrm{C}_{44}(\mathrm{GPa})$ \\
\hline AlN & $(001)$ & 2.42 & 3230 & 345 & 125 & 120 & 395 & 118 \\
\hline Mo & $(111)$ & 0.78 & 10228 & 463 & 157 & 157 & 463 & 109 \\
\hline
\end{tabular}

TABLE 1. Parameters used in the plate wave mode calculation.

The thickness of the AlN layer used in the calculation was chosen so that the series resonance (thickness expansion mode in the normal direction) was at the experimentally determined frequency of $875 \mathrm{MHz}$. This results in a total thickness for the plate of 3.98 $\mu \mathrm{m}$, which compares reasonably well with the experimentally determined total thickness of $3.80 \mu \mathrm{m}$. Figure 4 shows that the main features of the measured dispersion curve are reproduced in the calculation and allow identification of the modes as the lowest four plate wave modes, S1, A1, S0, A0. However, a discrepancy is seen in the wavenumber dependence of the curves that is due to the oversimplified modeling approach used for the calculation. First, the piezoelectric effect in AlN has not been taken into account. Second, the plate is not infinite in lateral extent with boundaries along both $\mathrm{X} \& \mathrm{Y}$ directions that must satisfy electrical and elastic boundary conditions, this can significantly affect the dispersion of the lower order modes [11].

\section{CONCLUSION}

FBAR measurements have been presented using a full-field imaging method that rapidly measures the acoustic amplitude and phase over the entire surface simultaneously. Detailed analysis of the images in the Fourier transform domain identifies the lateral modes observed as S0, S1 and A0, A1 plate waves. A comparison between the measured and calculated dispersion curves agrees quantitatively very well but still illustrates the need for developing a more complete model including piezoelectricity and finite size effects.

\section{ACKNOWLEDGMENTS}

This work was sponsored by the U.S. Department of Energy, Office Basic Energy Sciences, Materials and Engineering Physics under DOE Idaho Operations Office Contract DE-AC07-99ID13727. The author thanks Agilent Technologies Inc. and particularly John D. Larson III for supplying the FBAR sample and characterization parameters. The author also is grateful for the continuing help of Dave Cottle, Rob Schley and John Walter at the INEEL for assisting in earlier phases of this work.

\section{REFERENCES}

1 Telschow, K. L. Deason, V. A. Cottle, D. L. and Larson III, J. D., "Full-field Imaging of Acoustic Motion at Nanosecond Time and Micron Length Scales,” in 2002 IEEE Ultrasonics Symposium Proceedings, Vol.1, D. Yuhas and S. Schneider, eds., IEEE, Piscataway, NJ. 601-604 (2002).

2 Telschow, K. L. Deason, V. A. Cottle, D. L. and Larson III, J. D., "UHF Acoustic Microscopic Imaging of Resonator Motion” in 2000 IEEE Ultrasonics Symposium Proceedings, Vol.1, S.C. Schneider, M. Levy, B. R. McAvoy eds., IEEE, Piscataway, NJ. 631-634 (2000). 
3 Telschow, K. L. Deason, V. A. Cottle, D. L. and Larson III, J. D., "Full-Field Imaging Of GHz Film Bulk Acoustic Resonator Motion,” IEEE Trans. Ultrason., Ferroelect., Freq. Contr. 50 (10), 1279-1285 (2003).

4 Telschow, K. L., Deason, V. A., Schley, R. S., Watson, S. M., and, "Direct Imaging of Lamb Waves in Plates using Photorefractive Dynamic Holography," J. Acoust. Soc. Am. 106 (5), 2578-2587 (1999).

5 FBAR resonators and electrical characterization were provided by John D. Larson III, Device Technologies Division, Agilent Technologies Inc, Palo Alto, CA

6 Auld, B. A., Acoustic Fields and Waves in Solids (R.E. Krieger, Malabar, Fl., 1990) Vol. I, 396-398.

7 Pensala, T., Makkonen, T., Vartiainen, J., Knuutila, J., Kaitila, J., Holmgren, O. and Salomaa, M. M., "Laser Interferometric Measurement of Lamb Wave Dispersion and Extraction of Material Parameters in FBARs,” in 2002 IEEE Ultrasonics Symposium Proceedings, Vol.1, S. C. Schneider, D. E. Yuhas eds., IEEE, Piscataway, NJ. 977-980 (2002).

8 Mukdadi, O., Datta, S. K., Telschow, K. L., and Deason, V. A., “Ultrasonic Guided Waves in Thin Orthotropic Layers: Theoretical Analysis and Dynamic Holographic Imaging Measurement,” IEEE Trans. Ultrason., Ferroelect., Freq. Contr. 48 (6) 15811593 (2001).

9 Tsubouchi, K., \& Mikishiba, N.,"Zero-Temperature-Coefficient SAW Devices on AlN Epitaxial Films,” IEEE Trans. Sonics Ultr. SU-32 (5), 634-644 (1985).

10 Dickinson, J. M. and Armstrong, P. E., J. Appl. Phys. 38, 602-606 (1967)

11 Mukdadi, O. M., Desai, Y. M., Datta, S. K., Shah, A. H. and Niklasson, A. J., "Elastic guided waves in a layered plate with rectangular cross section,” J. Acoust. Soc. Am 112 (5), 1766-1779 (2002). 